\title{
Protective effect of tetrahydroxystilbene glucoside on cardiotoxicity induced by doxorubicin in vitro and in vivo
}

\author{
Shao-hui ZHANG, Wen-quan WANG, Jia-ling WANG* \\ Department of Pharmacology, Institute of Basic Medical Sciences, Tongji Medical College, Huazhong University of Science and Tech- \\ nology, Wuhan 430030, China
}

\begin{abstract}
Aim: To test the effect of 2,3,5,4'-tetrahydroxystilbene-2-O- $\beta$-D-glucoside (THSG) on doxorubicin (DOX)-induced cardiotoxicity. Methods: We used neonate rat cardiomyocytes and an acute mouse model of DOX-induced cardiotoxicity to examine the protective effect of THSG.

Results: In the mouse model, administration of THSG significantly reduced DOX-induced cardiotoxicity, including animal mortality, histopathological changes, and levels of serum creatine kinase (CK) and lactate dehydrogenase (LDH). Moreover, THSG was able to attenuate the increased malondialdehyde (MDA) and decreased reduced glutathione (GSH) caused by DOX. In in vitro studies, THSG 10-300 $\mu \mathrm{mol} / \mathrm{L}$ ameliorated DOX-induced cardiomyocyte apoptosis in a concentration-dependent manner. Further studies showed that THSG inhibited reactive oxygen species (ROS) generation and prevented DOX-induced loss of mitochondrial membrane potential, caspase-3 activation and upregulation of Bax protein expression. We observed a protective response against damage after DOX treatment. The level of Bcl-2 protein was increased. Additionally, THSG inhibited a DOX-induced $\left[\mathrm{Ca}^{2+}\right]$ increase.

Conclusion: These results showed that THSG protected against DOX-induced cardiotoxicity by decreasing ROS generation and intracellular $\left[\mathrm{Ca}^{2+}\right]$ and by inhibiting apoptotic signaling pathways.
\end{abstract}

Keywords: 2,3,5,4'-tetrahydroxystilbene-2-0- $\beta$-D-glucoside; doxorubicin; apoptosis; reactive oxygen species

Acta Pharmacologica Sinica (2009) 30: 1479-1487; doi: 10.1038/aps.2009.144

\section{Introduction}

Doxorubicin (also called adriamycin) is an anthracycline antibiotic that has been used for more than 30 years for the treatment of a wide variety of cancers. However, severe cardiomyopathy and heart failure have been observed in DOXtreated cancer patients ${ }^{[1]}$, which limits clinical chemotherapy. It is widely accepted that oxidative stress and the production of free radicals are involved in DOX-induced cardiotoxicity ${ }^{[2-7]}$. The quinone moiety of DOX is prone to the generation of oxygen radicals through an enzymatic mechanism utilizing mitochondrial respiratory chain and non-enzymatic pathways that incorporate iron ${ }^{[8,9]}$. DOX-induced cardiomyocyte apoptosis has been further reported in specific conditions ${ }^{[10,11]}$, and mitochondrial dysfunction has a significant role in contributing to DOX-induced apoptosis ${ }^{[12-14]}$.

Polygonum multiflorum Thunb, a traditional Chinese medicinal herb, has been used for thousands of years as a tonic

* To whom correspondence should be addressed.

E-mail wangtjmu@hotmail.com

Received 2009-07-04 Accepted 2009-08-31 and anti-aging agent. THSG is one of the active components extracted from Polygonum multiflorum Thunb and has been shown to exhibit strong antioxidant activity in vitro ${ }^{[15]}$. Researchers have shown that THSG can diminish peroxidation levels in the brain in a mouse model of Alzheimer's disease ${ }^{[16]}$ or cerebral ischemia-reperfusion ${ }^{[17]}$. Structurally, THSG belongs to the hydroxystilbene group (Figure 1), and its structure is similar to that of resveratrol extracted from red wine, which has significant protective effects on cardiomyocyte injury. Based on these previous reports, we envisioned that THSG might protect the heart from DOX-induced injury.

In the present study we used neonatal rat cardiomyocytes in vitro and an acute mouse model of DOX-induced cardiotoxicity to examine the protective effects of THSG as well as the underlying mechanisms.

\section{Materials and methods}

Drugs and chemicals

Doxorubicin was provided by Pharmacia Italia SpA. THSG was provided by the Department of Pharmacology, Tongji Medical College, Huazhong University of Science 


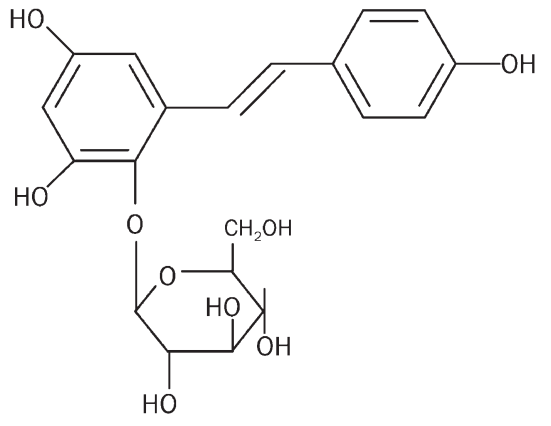

Figure 1. The chemical structure of THSG.

and Technology (China) with a purity of 99\% (HPLC method $)^{[18]}$. Anti-caspase-3, anti-Bax, anti-Bcl-2, and antiactin were purchased from Santa Cruz Biotechnology. The Super Signal West Pico chemiluminescence substrate was obtained from PIERCE Biotechnology. The TUNEL assay kit was obtained from Wuhan Boster Biological Technology. Methylthiazolyldiphenyl-tetrazolium bromide (MTT), 5,5' ,6,6'tetrachloro-1,1',3,3' -tetraethylbenzimidazolocarbocyanine iodide (JC-1), and 2',7'-dichlorodihydrofluorescein diacetate (DCFH-DA) were obtained from the Beyotime Institute of Biotechnology. Fluo 3-AM was purchased from SigmaAldrich. MDA, GSH, LDH, and CK assay kits were purchased from the Nanjing Jiancheng Bioengineering Institute.

\section{Animals and treatments}

Kunming male mice of inbred strain, 20-24 g body weight, were randomly divided into three groups consisting of 20 animals each. The animals were housed under standard conditions and were given free access to food and tap water. DOX was intraperitoneally (ip) injected at a dose of $15 \mathrm{mg} / \mathrm{kg}$, which is well-documented to induce cardiotoxicity in mice. THSG was administered at a dose of $0.1 \mathrm{~g} \cdot \mathrm{kg}^{-1} \cdot \mathrm{d}^{-1}$, which was selected on the basis of previous studies ${ }^{[19]}$. The DOX group received a single dose of DOX following treatment with isotonic saline for five days. The group that was administered with THSG was treated for two days prior to a single dose of DOX and for five days after. The control group received a single dose via ip injection of isotonic saline following treatment with isotonic saline for five days. All animal care and experimental protocols complied with the Animal Management Rules of the Health Ministry of the People's Republic of China (document № 55, 2001).

\section{Sample collection and biochemical assays}

At the end of the treatment, all animals were decapitated, and blood samples were collected. The plasma was removed immediately for the LDH and CK assay. The heart was quickly isolated, blotted dry on filter paper, and weighed. Thereafter, a $10 \%$ homogenate of the heart was made in icecold saline to determine MDA and GSH concentrations. The reagent kits were used to determine the biochemical indicators.

\section{Histopathological examinations}

The tissues were fixed in $10 \%$ formalin, routinely processed and embedded in paraffin. Sections were cut at 5 - $\mu \mathrm{m}$ thicknesses and stained with hematoxylin and eosin. The sections were then viewed under a light microscope for histopathological changes.

\section{Isolation and cultivation of rat cardiomyocytes}

Primary cultures of cardiomyocytes from neonatal (2-4 days old) Wistar rats were prepared according to the published method $^{[20]}$. The cell suspension was adjusted to a density of $3 \times 10^{5}-5 \times 10^{5}$ cells $/ \mathrm{mL}$ and seeded in 6 - or 96 -well plastic plates. Forty-eight hours after seeding, the attached cells began to contract spontaneously. The experiments were performed between three and four days after cultivation.

\section{Cell viability assay}

The cell viability was measured using the MTT assay, which is based on the conversion of MTT to formazan crystals by mitochondrial dehydrogenase. For our purpose, cells were seeded in 96-well plates and then received $1 \mu \mathrm{mol} / \mathrm{L}$ DOX either alone or with THSG $(3 \sim 300 \mu \mathrm{mol} / \mathrm{L})$ pretreatment $2 \mathrm{~h}$ before. After incubation for up to $24 \mathrm{~h}$, MTT solution (5 $\mathrm{mg} / \mathrm{mL}$ in D-Hanks' solution) was added to the 96 -well plates, and the cells were allowed to incubate for $4 \mathrm{~h}$ at $37^{\circ} \mathrm{C}$. After the medium was removed and regular medium was added to prevent the drugs from reacting directly with MTT, the cells and formazan were dissolved by adding dimethylsulfoxide (DMSO), and the light absorbance was measured at $490 \mathrm{~nm}$ in a microtiter plate reader.

\section{Cardiomyocyte apoptosis}

Cardiomyocyte apoptosis was evaluated via the terminal deoxynucleotidyl transferase-mediated dUTP nick-end labeling (TUNEL) method according to the manufacturer's instructions. Labeled DNA was visualized with peroxidaseconjugated anti-digoxigenin antibody with 3,3'-diaminobenzidene as the chromagen. The quantification of apoptosis was determined by counting TUNEL-positive cardiomyocyte nuclei from ten random fields per section and was expressed as a percentage of total nuclei.

\section{Determination of ROS generation}

To measure intracellular ROS formation, the fluorescent probe DCFH-DA was used. Briefly, the cardiomyocytes attached to plates were loaded with $10 \mu \mathrm{mol} / \mathrm{L}$ DCFH-DA in serumfree medium at $37^{\circ} \mathrm{C}$ for $20 \mathrm{~min}$, and the plates were washed three times with serum-free medium and used for the treatment with various drugs. Fluorescence of ROS in cardiomyocytes was recorded with a confocal scanning laser microscope (FV500, Olympus, Japan) and analyzed with PathVision imaging software.

\section{Measurement of mitochondrial membrane potential}

The mitochondrial membrane potential was studied using the probe JC-1, which exists either as a green fluorescent monomer 
at depolarized membrane potentials or as a red fluorescent J-aggregate at hyperpolarized membrane potentials. Cells were loaded by changing the culture medium to phosphatebuffered saline (PBS) containing $1 \mu \mathrm{mol} / \mathrm{L} \mathrm{JC}-1$ for $20 \mathrm{~min}$ at $37^{\circ} \mathrm{C}$. The fluorescent dye was excited at $490 \mathrm{~nm}$, and the fluorescence intensities of both monomer and aggregated JC-1 molecules were recorded at $590 \mathrm{~nm}$ with the confocal scanning laser microscope. The ratio of mitochondrial aggregates (red) to the monomeric form of JC-1 (green) was analyzed with PathVision imaging software.

\section{Western blot analysis}

After the treatment, the cells were washed twice with cold PBS and solubilized in lysis buffer. Fifty micrograms of protein sample were separated by $12 \%$ SDS-polyacrylamide gel electrophoresis and transferred onto a nitrocellulose membrane. The membrane was incubated with anti-caspase 3, anti-Bax, anti-Bcl-2 and anti-actin as primary antibodies in 5\% nonfat milk in PBS with $0.1 \%$ Tween 20, followed by incubation with horseradish peroxidase-conjugated IgG as the secondary antibody. The chemiluminescence reaction was carried out using an ECL kit.

\section{Determination of $\left[\mathrm{Ca}^{2+}\right]_{\mathrm{i}}$}

Briefly, the cardiomyocytes attached to plates were loaded with $5 \mu \mathrm{mol} / \mathrm{L}$ Fluo 3-AM in Krebs-Henseleit (KH) buffer at $37^{\circ} \mathrm{C}$ for $30 \mathrm{~min}$. The plates were washed three times with $\mathrm{KH}$ buffer and treated with various drugs. THSG at different concentrations was pre-incubated at $37^{\circ} \mathrm{C}$ for $30 \mathrm{~min}$ prior to treatment with DOX $(1 \mu \mathrm{mol} / \mathrm{L})$. The $\left[\mathrm{Ca}^{2+}\right]_{\mathrm{i}}$ signal in cardiomyocytes was measured for 5 min with a confocal scanning laser microscope.

\section{Statistical analysis}

Data were expressed as mean \pm SD based on the data derived from multiple independent experiments. Statistical differences were determined by ANOVA followed by a Student's $t$-test. $P$ values below 0.05 were considered statistically significant.

\section{Results}

Effects of DOX and THSG treatment on animal body weight and survival

DOX treatment for five days reduced the animals' body weights compared with the control $(21.8 \pm 3.6 \mathrm{~g}$ vs $25.6 \pm 2.6 \mathrm{~g}$, $P<0.01)$. Treatment with THSG did not increase the animal's body weight (22.5 $\pm 2.8 \mathrm{~g}, P>0.05$, compared with DOX alone). There was no difference in the heart weight/body weight ratio among the three groups $(4.5 \pm 0.6 \mathrm{mg} / \mathrm{g}$ in control group, $4.3 \pm 0.7 \mathrm{mg} / \mathrm{g}$ in DOX treatment and $4.3 \pm 0.4 \mathrm{mg} / \mathrm{g}$ in THSG treatment). At the end of the treatment period, all animals in the control group were alive. However, only $70 \%$ of the animals in the DOX-alone group survived. THSG-treated animals had a compromised survival rate of $85 \%$ compared with DOX alone (log rank test, $P>0.05$ ).

\section{Effects of DOX and THSG treatment on serum LDH and CK and heart MDA and GSH}

The activities of serum LDH and CK have been widely used clinically as parameters for the diagnosis of cardiac diseases. As shown in Table 1, DOX alone induced significant increases in serum LDH and CK levels compared with the control, and these increases were effectively attenuated by THSG treatment. DOX treatment also led to a significant increase in MDA and a depletion in GSH compared with that of the control group. The combination of THSG and DOX provided marked normalization in MDA and GSH concentrations compared with the DOX-alone group.

Table 1. The effect of THSG $\left(0.1 \mathrm{~g}^{\mathrm{kg}} \mathrm{k}^{-1} \cdot \mathrm{d}^{-1}\right)$ on DOX-induced changes in serum LDH, CK and oxidative biochemical parameters (MDA and GSH). $n=12$ in each group; ${ }^{\circ} P<0.01$ vs Control; ${ }^{e} P<0.05,{ }^{f} P<0.01$ vs DOX alone.

\begin{tabular}{lrcl}
\hline & Control & DOX & DOX+THSG \\
\hline LDH $(\mathrm{U} / \mathrm{mL})$ & $3.87 \pm 0.40$ & $6.00 \pm 0.27^{\circ}$ & $4.86 \pm 0.20^{\mathrm{e}}$ \\
CK $(\mathrm{U} / \mathrm{mL})$ & $1.55 \pm 0.49$ & $3.02 \pm 0.28^{\circ}$ & $1.94 \pm 0.4^{\mathrm{e}}$ \\
MDA (nmol/mg protein) & $1.15 \pm 0.30$ & $4.17 \pm 0.91^{\circ}$ & $2.32 \pm 0.76^{\mathrm{f}}$ \\
GSH (mg/g protein) & $24.43 \pm 6.57$ & $13.65 \pm 3.46^{\circ}$ & $18.31 \pm 3.27^{\mathrm{f}}$ \\
\hline
\end{tabular}

\section{THSG attenuated DOX-induced heart histological changes}

Normal heart histological findings were seen in the control group (Figure 2A). However, there were histological changes in the DOX group (Figure $2 \mathrm{~B}-2 \mathrm{C}$ ), including the presence of marked interstitial edema, focal subendocardial hemorrhage, and destruction or loss of cardiac muscle fibers, all of which was qualitatively recognized as DOX-induced cardiac damage. The lesions were significantly reduced in the group treated with THSG compared with the DOX group (Figure 2D).

THSG increased the viability of DOX-treated cells

The effect of THSG on the cytotoxicity of DOX was detected by the MTT assay. As shown in Figure 3, after treatment with $300 \mathrm{mmol} / \mathrm{L}$ THSG for $24 \mathrm{~h}$, no obvious effect was seen, as the cell viability was $102.5 \% \pm 5.9 \%$ ( $P>0.05$, compared with the control). DOX alone markedly affected cell survival, as the rate of cell survival after exposure to $1 \mu \mathrm{mol} / \mathrm{L}$ DOX for $24 \mathrm{~h}$ was about $72 \%$. In the presence of THSG, cell viability was significantly enhanced in a concentration-dependent manner. With the exception of the $3 \mu \mathrm{mol} / \mathrm{L}$ THSG group, all THSG groups had significant differences compared with the DOXalone group, indicating that THSG inhibited DOX-induced cell death.

\section{THSG inhibited DOX-induced apoptosis}

Compared with the control cells, more apoptotic nuclei were identified in the cells grown in medium with DOX (Figure $4 \mathrm{~A}-4 \mathrm{~B})$, and the rate of positive cells was $34.2 \% \pm 4.5 \%$ by quantitative analysis. As shown in Figure 4C,D, E, treatment with 10-300 $\mu \mathrm{mol} / \mathrm{L}$ THSG decreased the number of apoptotic nuclei after DOX incubation in a concentration-dependent 

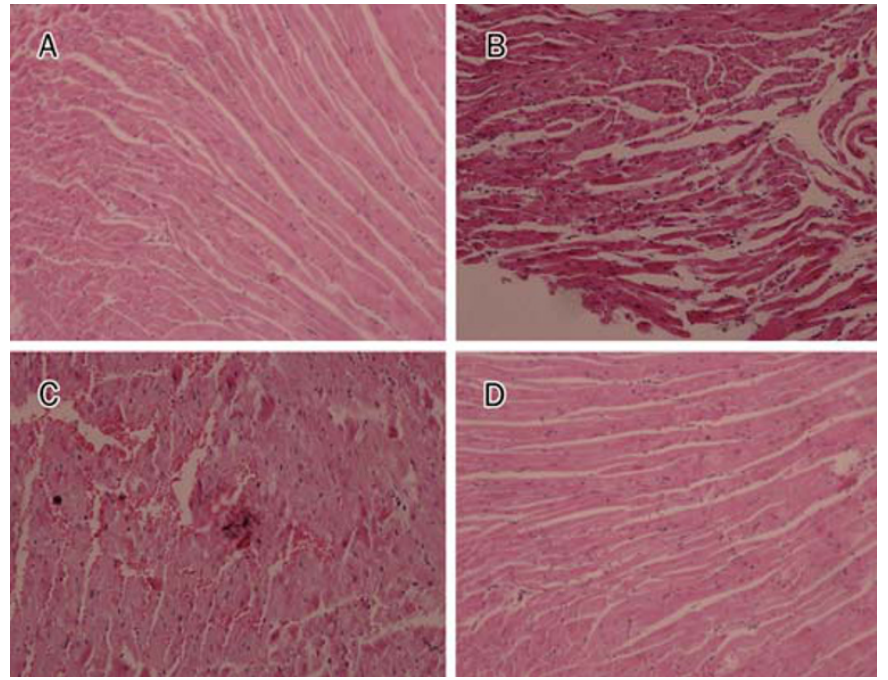

Figure 2. Histologic evaluation of the cardiac tissue corresponding to untreated DOX group and THSG treated group $(H \& E, \times 10)$. (A) control; $(B, C)$ DOX alone; (D) treatment with THSG.

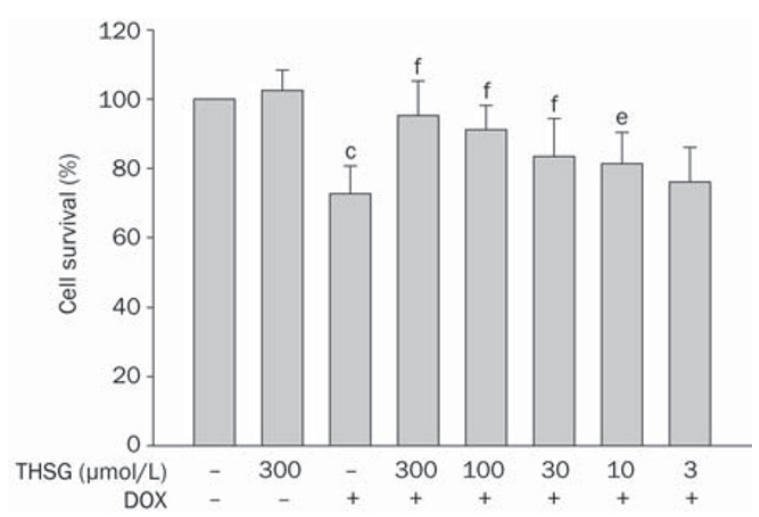

Figure 3. THSG enhanced the viability of DOX $(1 \mu \mathrm{mol} / \mathrm{L})$ treated neonate rat cardiomyocytes by MTT assay. ${ }^{\circ} P<0.01$, compared with control; ${ }^{\mathrm{e}} P<0.05,{ }^{\mathrm{f}} P<0.01$, compared with DOX alone. The data are representative of three independent experiments.

manner, and all groups had significant differences compared with the DOX-alone group, which was consistent with the results obtained from the MTT assay. These results confirmed that apoptosis of cardiomyocytes could be induced by DOX and that THSG could attenuate DOX-induced apoptosis.

\section{THSG prevented DOX-induced loss of mitochondrial membrane potential}

Nearly all cells were well-spread and exhibited red or orange fluorescence in untreated culture (Figure 5A). By contrast, treatment with DOX for $24 \mathrm{~h}$ (Figure 5B) demonstrated multiple rounded cells, a majority of which fluoresced green exclusively, indicating loss of mitochondrial membrane potential. The cells pretreated with THSG appeared similar to the control cells, with spread cells exhibiting red or orange fluorescence (Figure 5C). Ratios of JC-1 aggregates/monomeric forms in
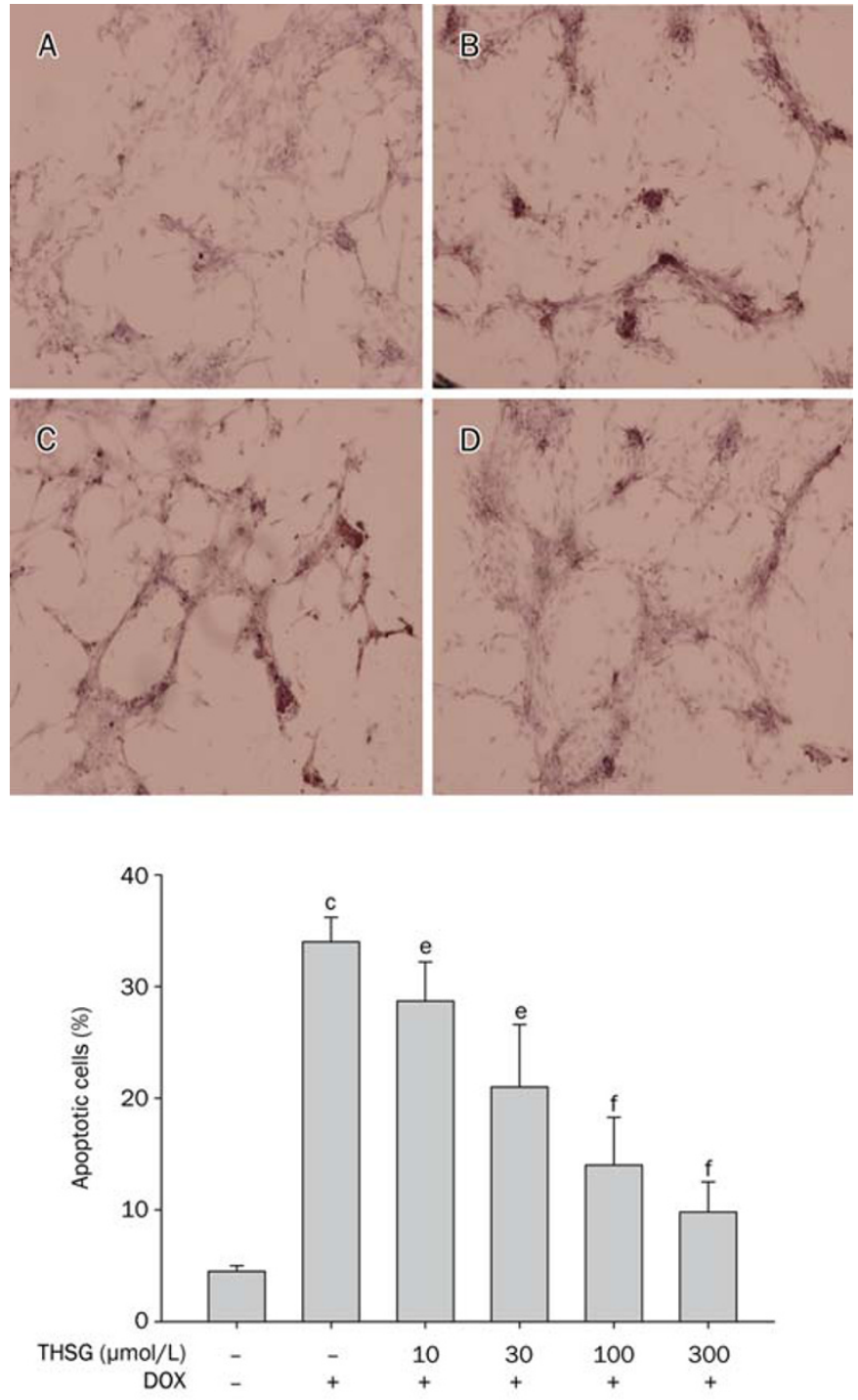

Figure 4. DOX induced cardiomyocytes apoptosis was inhibited by THSG. Cultured cells were untreated (negative control: $A$ ) or treated with 1 $\mu \mathrm{mol} / \mathrm{L}$ DOX for $24 \mathrm{~h}$ without (B) or with $10 \mu \mathrm{mol} / \mathrm{L}$ (C) and $300 \mu \mathrm{mol} / \mathrm{L}$ (D) THSG pretreated for $2 \mathrm{~h}$. Quantitative presentation of TUNEL assay (E). ${ }^{\mathrm{C}} P<0.01$, compared with control; ${ }^{\mathrm{e}} P<0.05,{ }^{\mathrm{f}} P<0.01$, compared with DOX alone. Results are means $\pm S D$ of three individual experiments.

all groups (Figure 5D) indicate that DOX-induced loss of the mitochondrial membrane potential was prevented by THSG.

\section{THSG inhibited DOX-induced ROS generation}

Treatment with $300 \mu \mathrm{mol} / \mathrm{L}$ THSG for $24 \mathrm{~h}$ had no effect on ROS levels in untreated cells (Figure 6A-6B). As shown in Figure $6 \mathrm{C}$, treatment of cells with DOX stimulated a significant increase in the ROS level. The increase in ROS production was significantly reduced by pretreatment with $300 \mu \mathrm{mol} / \mathrm{L}$ THSG for $2 \mathrm{~h}$ (Figure 6D). A direct comparison of ROS levels in all groups is summarized in Figure 6E. These results indicate that THSG attenuates DOX-induced intracellular ROS accumulation. 

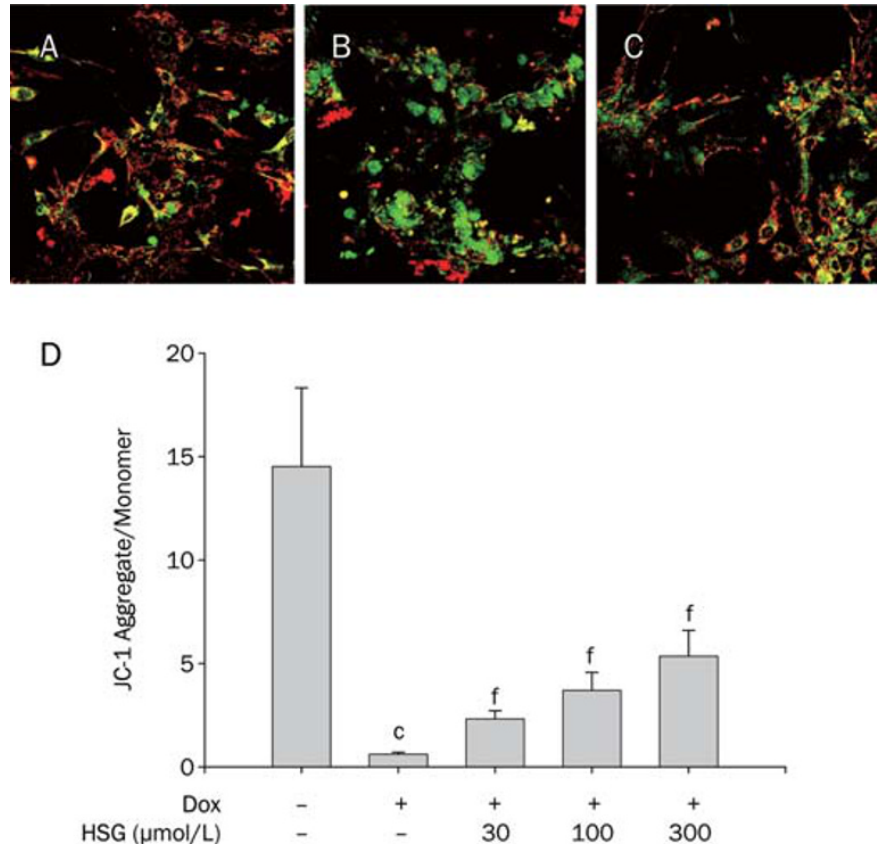

Figure 5. DOX induced loss of mitochondrial membrane potential was prevented by THSG. Cultured cells were untreated $(A)$ or treated with 1 $\mu \mathrm{mol} / \mathrm{L}$ DOX for $24 \mathrm{~h}$ without (B) or with $300 \mu \mathrm{mol} / \mathrm{L}$ THSG pretreated for $2 \mathrm{~h}(\mathrm{C})$. Quantitative presentation of JC-1 assay (D). ${ }^{\mathrm{C}} \mathrm{P}<0.01 \mathrm{vs}$ control; ${ }^{\mathrm{f}} P<0.01$ vs DOX alone. Results are means \pm SD of triplicate determinations.

\section{Effects of THSG and DOX on the expression of apoptosis-related proteins}

To examine the molecular mechanism of THSG against DOXinduced apoptosis, the effects of THSG on DOX-mediated caspase- 3 activation and the changes in the protein levels of Bax and Bcl-2 were determined by Western blot assay. As shown in Figure 7, treatment of the cells with $1 \mu \mathrm{mol} / \mathrm{L}$ DOX for 24 $\mathrm{h}$ induced the cleavage of procaspase- $3(32 \mathrm{kDa})$ to its $17-\mathrm{kDa}$ subunits. However, cleavage of procaspase- 3 to its subunits was inhibited by THSG in a dose-dependent manner. Preincubation with $300 \mu \mathrm{mol} / \mathrm{L}$ THSG for $2 \mathrm{~h}$ completely eliminated caspase- 3 activation in DOX-treated cells, indicating that DOX activates caspase- 3 , and this step is prevented by THSG.

Bcl-2 family proteins have an important role in regulating cytochrome $c$ release and caspase- 3 activation. Bcl-2, an antiapoptotic protein, prevents the release of cytochrome $c$ from mitochondria, whereas Bax, a pro-apoptotic protein, promotes release of cytochrome $c$ from mitochondria. As shown in Figure 7, treatment of cells with DOX induced a marked increase in the protein level of Bcl-2. Pre-incubation with THSG made this potentially adaptive response more outstanding. Compared with the control, the abundance of Bax was increased after the addition of DOX, indicating that Bax is involved in the apoptotic signaling induced by DOX treatment. After pretreatment with THSG, Bax protein induction was reduced in a dose-dependent manner (Figure 7B). These results suggest that THSG might attenuate the apoptotic effects of DOX partly by inhibiting caspase- 3 activation, Bax induction and improv-
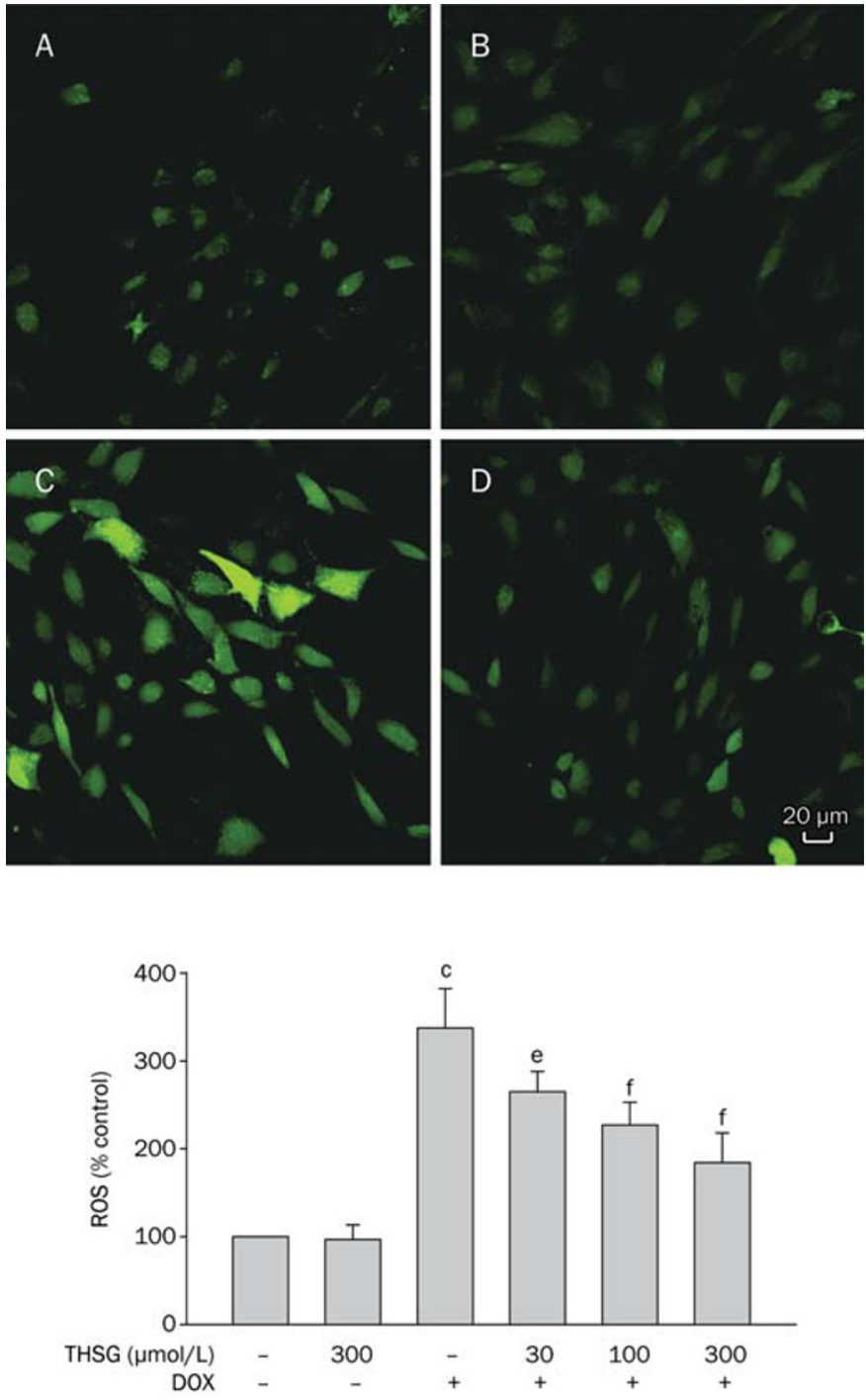

Figure 6. DOX-mediated ROS generation was inhibited by THSG. (A) control; (B) $300 \mu \mathrm{mol} / \mathrm{L}$ THSG alone; (C) $1 \mu \mathrm{mol} / \mathrm{L}$ DOX treatment for $24 \mathrm{~h}$ alone; (D) $300 \mu \mathrm{mol} / \mathrm{L}$ THSG pretreated for $2 \mathrm{~h}$. (E) Quantitative results (green fluorescence area) are expressed as \% of values found in cells untreated. ${ }^{\mathrm{c}} P<0.01$, compared with control; ${ }^{\mathrm{f}} P<0.01$, compared with DOX alone. Data are means $\pm \mathrm{SD}$ of three independent experiments.

ing Bcl-2 protein expression.

\section{THSG inhibited the DOX-induced $\left[\mathrm{Ca}^{2+}\right]_{\mathrm{i}}$ increase}

As shown in Figure 8A, treatment of cardiomyocytes with DOX greatly increased the $\left[\mathrm{Ca}^{2+}\right]_{i}$; the $\left[\mathrm{Ca}^{2+}\right]_{\mathrm{i}}$ increase was very quick and did not reach a plateau at $5 \mathrm{~min}$. Pretreatment with $30 \mu \mathrm{mol} / \mathrm{L}$ THSG inhibited DOX-induced elevation of $\left[\mathrm{Ca}^{2+}\right]_{\mathrm{i},}$ and a plateau was reached in approximately $2 \mathrm{~min}$ (Figure 7B). Treatment with 100 and $300 \mu \mathrm{mol} / \mathrm{L}$ THSG nearly maintained basal $\left[\mathrm{Ca}^{2+}\right]_{i}$ levels after DOX was added (Figure 7C-7D). Quantitative data are summarized in Figure 7E. These results indicate that DOX increased the $\left[\mathrm{Ca}^{2+}\right]_{i}$, and this could be prevented with THSG. 

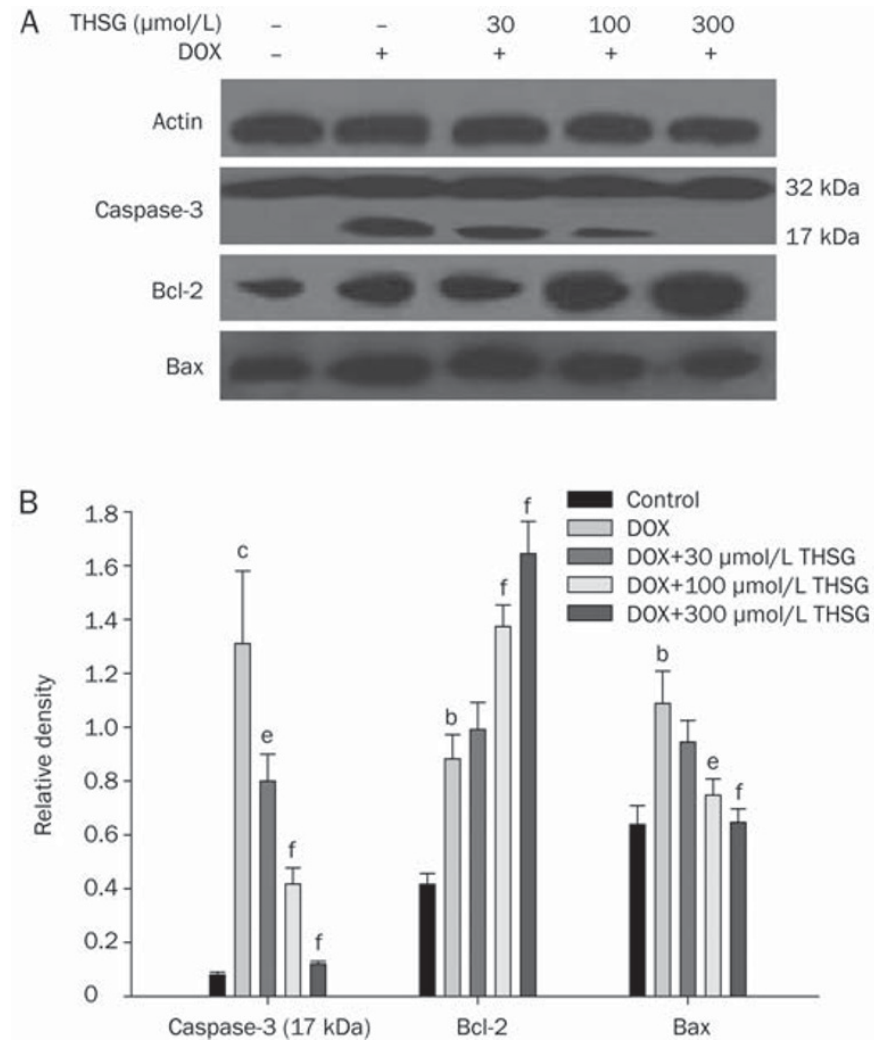

Figure 7. Effects of THSG and DOX (1 $\mu \mathrm{mol} / \mathrm{L})$ on the expression of apoptosis-related proteins. (A) THSG ihibited DOX-induced caspase-3 activation and Bax protein expression but increased DOX-induced $\mathrm{Bcl}-2$ protein expression. (B) Bar graph shows densitometric quantification from 3 individual experiments, data are normalized against the actin level for each sample. ${ }^{\mathrm{b}} P<0.05,{ }^{\mathrm{c}} P<0.01$, compared with control; ${ }^{\mathrm{e}} P<0.05$, ${ }^{\mathrm{f}} P<0.01$, compared with DOX alone.

\section{Discussion}

DOX-induced cardiotoxicity manifests itself in acute, subacute and chronic forms ${ }^{[21]}$. In the current studies of DOX cardiotoxicity, the single high-dose model and the low-dose chronic model are both widely used, which provides valuable biological insights into DOX-induced cardiac injury. For the single high-dose model, the dosage is equivalent to a high-dose single injection in cancer patients ${ }^{[22]}$. In the current study, we applied the single high-dose model and investigated whether THSG protects the heart from acute DOX toxicity. The results showed that THSG greatly decreased the DOX-associated elevation of serum LDH and CK activities, two nonspecific but widely used cardiac injury markers. Furthermore, THSG attenuated DOX-induced animal mortality and histology changes. These findings indicate that THSG is a potential protective agent against DOX injury. It is known that enhanced oxidative stress and antioxidant deficit play significant parts in DOX-induced cardiomyopathy and congestive heart failure ${ }^{[23]}$. The findings of the present study conform to this understanding, as elevated cardiac lipid peroxidation, accompanied by deteriorating antioxidant status, was evident in the DOX group. However, THSG administration prevented an upsurge in oxidative stress, as shown by the low level of tissue lipid peroxides in the treated group. The restoration of the normal levels of GSH antioxidant molecules in the THSGtreated group highlight the protection rendered by THSG in combating the oxidative insult. This finding indicates that the protective effect of THSG involves its antioxidant activity and radical scavenging.

Another important mechanism of DOX-induced cardiotoxicity is apoptosis. In regard to the mitochondrial pathway, several studies have shown that DOX induces apoptosis by favoring cytochrome $c$ release and the consequent formation of the apoptotic complex through up-regulation of $\mathrm{Bax}^{[10,24]}$ or down-regulation of $\mathrm{Bcl}-2^{[25,26]}$. The results obtained from this study confirmed that apoptosis of cardiomyocytes could be induced by DOX because DOX induced caspase- 3 activation and increased Bax expression. THSG was able to inhibit DOXinduced apoptosis in the cardiomyocytes. This inhibition correlated with the inhibitory effect of THSG on DOX-induced caspase- 3 activation and increased Bax protein expression. Of note, we observed that DOX induced a marked increase in the protein level of Bcl-2, and THSG treatment promoted this adaptive response. Perhaps this increase is involved in some protective responses that occur against subsequent damage after DOX treatment.

DOX was able to enhance the formation of ROS in heart tissue. Excessive production of ROS may induce cell damage via apoptosis in any cell type, and such effects can be blocked by a wide variety of antioxidants ${ }^{[27,28]}$. In line with this notion, we observed that DOX-induced intracellular ROS accumulation was attenuated by THSG, as revealed by reduced distribution of the DCFH-DA fluorescent dye in cells pretreated with THSG. Many reports have demonstrated that cytochrome c-dependent caspase- 3 activation is an important mechanism responsible for ROS-induced apoptosis in vivo and in vitro ${ }^{[29]}$. We found that DOX activated caspase-3, and this activation could be prevented by THSG, indicating that THSG reduced DOX-induced apoptosis through the inhibition of caspase-3 activation, which is, at least in part, induced by ROS.

Apoptotic responses may reflect direct opening of the mitochondrial permeability transition by $\mathrm{O}_{2}^{-}$and $\mathrm{H}_{2} \mathrm{O}_{2}$ formed during the redox cycling of $\mathrm{DOX}^{[14,30]}$. Accumulation of the more lipophilic 7-deoxyaglycone of DOX in the inner mitochondrial membrane greatly enhances electron deviation from the regular respiratory chain pathway to oxygen, leading to an increased formation of $\mathrm{O}_{2}^{-}$and $\mathrm{H}_{2} \mathrm{O}_{2}$ and consequent amplification of mitochondrial dysfunction ${ }^{[31]}$. In the current study, where cardiomyocytes were exposed to DOX, rapid depolarization of the mitochondrial membrane potential was also observed, which is indicative of mitochondrial dysfunction; this dysfunction was alleviated by pretreatment with THSG. Taken together, the present study demonstrates that THSG suppresses DOX-induced cardiomyocyte apoptosis through inhibition of the mitochondrial cytochrome $c$ release-mediated apoptotic pathway, which is triggered by the increased levels of ROS generated by DOX.

Although the theory has received less attention than ROS, 
A Dox alone

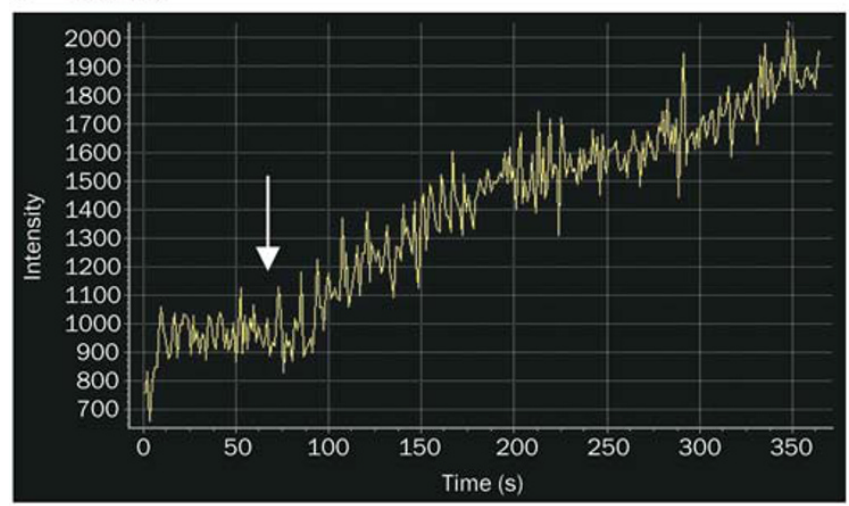

B $\mathrm{DOX}+30 \mu \mathrm{mol} / \mathrm{L}$ THSG pretreatment

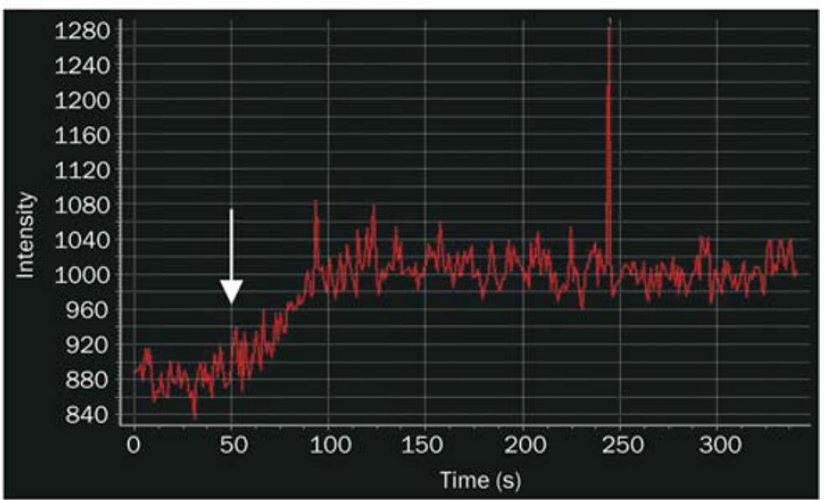

D DOX $+300 \mu \mathrm{mol} / \mathrm{L}$ THSG pretreatment

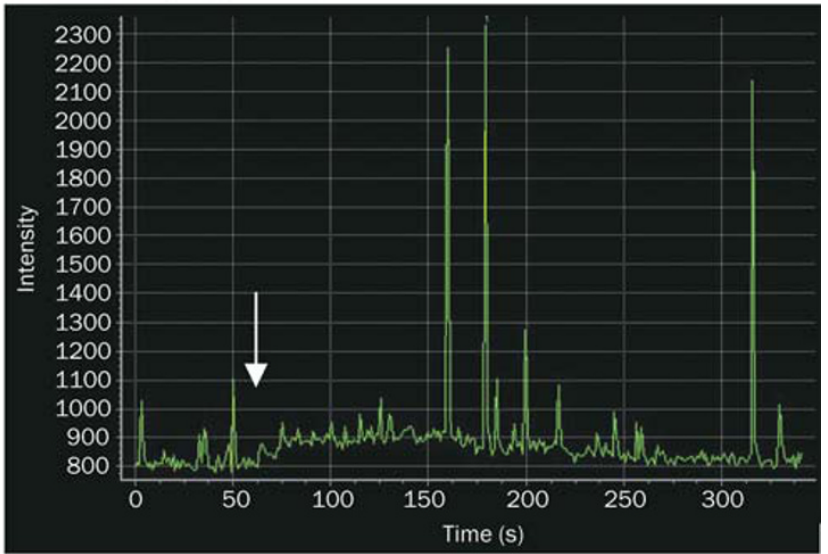

\section{Dox $+100 \mu \mathrm{mol} / \mathrm{L}$ THSG pretreatment}
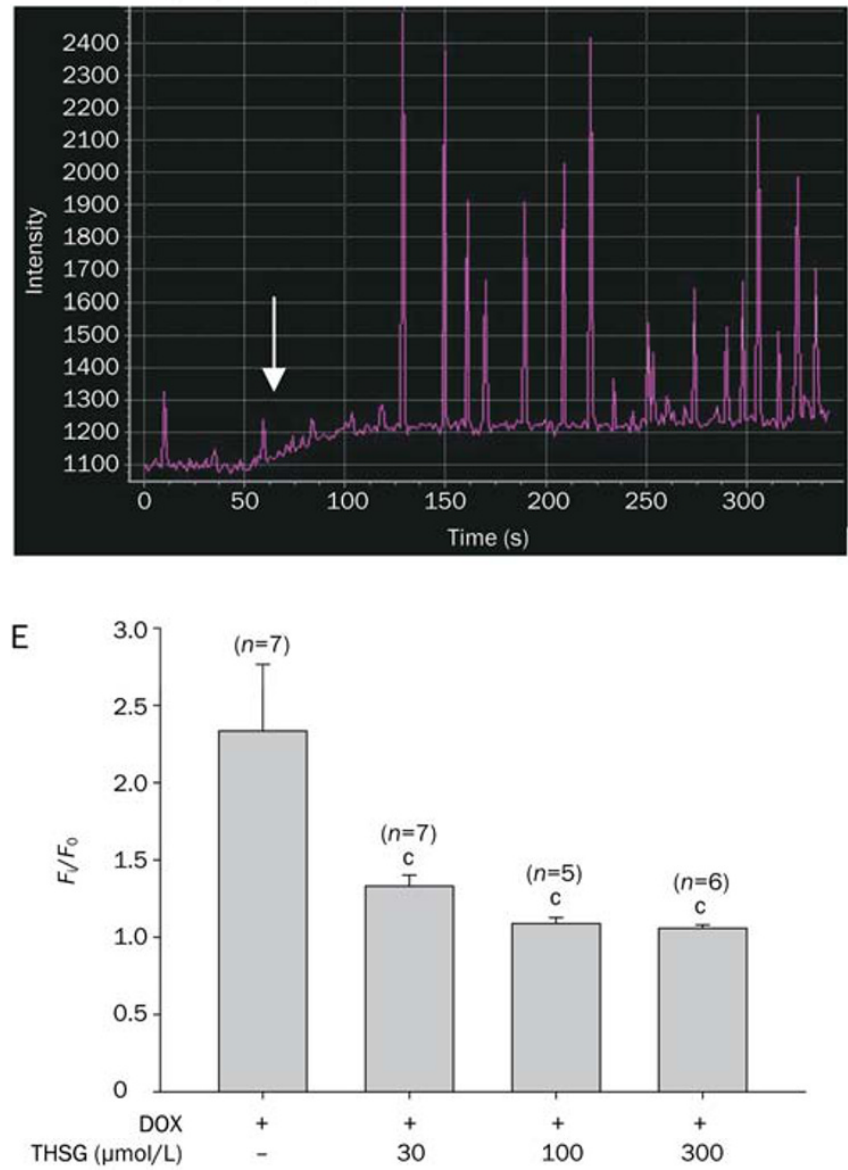

a number of studies have suggested that DOX-mediated alteration of $\mathrm{Ca}^{2+}$ homeostasis is one possible mechanism of cardiotoxicity. DOX increases the probability of sarcoplasmic reticulum (SR) calcium release channels being open ${ }^{[32,33]}$ thereby inhibiting the $\mathrm{Na}^{+}-\mathrm{Ca}^{2+}$ exchanger ${ }^{[34]}$ or activating L-type cardiac calcium channels ${ }^{[35]}$. In addition, an increase in the concentration of intracellular $\mathrm{Ca}^{2+}$ initiates a critical step for apoptosis by promoting ROS generation ${ }^{[36]}$ and modulating a transition in mitochondrial permeability by opening permeability transition pores, thereby releasing cytochrome $c^{[37,38]}$. Moreover, there is a close link between DOX-
Figure 8. Effects of THSG on $\left[\mathrm{Ca}^{2+}\right]_{\mathrm{i}}$ in DOX $(1 \mu \mathrm{mol} / \mathrm{L})$-treated cardiomyocytes. Cultured cells were pretreated with THSG at the concentration of 30 (B), 100 (C) and $300 \mu \mathrm{mol} / \mathrm{L}$ (D) or without THSG (A) for $30 \mathrm{~min}$ before DOX treatment. An arrow indicates the time point at where DOX was added. Quantitative data are expressed ratio of $F_{i} / F_{0}(E) .{ }^{c} P<0.01$, compared with DOX alone.

induced ROS generation and an increase in $\left[\mathrm{Ca}^{2+}\right]_{i}$, and a DOXinduced $\left[\mathrm{Ca}^{2+}\right]_{\mathrm{i}}$ increase is inhibited by pretreatment with antioxidants ${ }^{[39]}$. Consistent with these observations, our studies demonstrate that DOX-mediated increases in $\left[\mathrm{Ca}^{2+}\right]_{\mathrm{i}}$ are reduced by treatment with THSG, which also inhibits DOXinduced ROS generation.

We also found that THSG itself might have therapeutic potential as a protective agent. A previous report has proven that THSG inhibits tumor growth and lung metastasis ${ }^{[40]}$. We also found that 1-100 $\mu \mathrm{mol} / \mathrm{L}$ of THSG inhibited MCF-7 cell proliferation by conspicuously depressing the PI3K/Akt sig- 
naling pathway (data not shown). Further research should focus on drug antagonism in combination with DOX to ascertain whether THSG increases the antitumor efficacy of DOX. In summary, THSG appears to be a promising drug to be used in combination with DOX to ameliorate the cardiotoxicity while increasing the clinical efficacy of DOX and improving patients' quality of life.

\section{Acknowledgments}

This study was supported by grants from the National Science Fund for Young Scholars of China (№ 30801418).

\section{Author contribution}

Shao-hui ZHANG and Jia-ling WANG designed and performed the research; Wen-quan WANG contributed new analytical tools and reagents; Shao-hui ZHANG analyzed the data and wrote the paper.

\section{References}

1 Singal PK, lliskovic N. Doxorubicin-induced cardiomyopathy. N Engl J Med 1998; 339: 900-5.

2 Kotamraju S, Konorev EA, Joseph J, Kalyanaraman B. Doxorubicininduced apoptosis in endothelial cells and cardiomyocytes is ameliorated by nitrone spin traps and ebselen. Role of reactive oxygen and nitrogen species. J Biol Chem 2000; 275: 33585-92.

3 Kang YJ, Chen Y, Epstein PN. Suppression of doxorubicin cardiotoxicity by overexpression of catalase in the heart of transgenic mice. J Biol Chem 1996; 271: 12610-6.

4 Kang YJ, Chen Y, Yu A, Voss-McCowan M, Epstein PN. Overexpresssion of metallothionein in the heart of transgenic mice suppresses doxorubicin cardiotoxicity. J Clin Invest 1997; 100: 1501-6.

5 Doroshow JH, Locker GY, Baldinge RJ, Myers CE. The effect of doxorubicin on hepatic and cardiac glutathione. Res Commun Chem Pathol Pharmacol 1979; 26: 285-95.

6 Doroshow $\mathrm{JH}$. Effect of anthracycline antibiotics on oxygen radical formation in rat heart. Cancer Res1983; 43: 460-72.

7 Yen HC, Oberley TD, Vichitbandha S, Ho YS, St Clair DK. The protective role of manganese superoxide dismutase against adriamycin-induced acute cardiac toxicity in transgenic mice. J Clin Invest 1996; 98 : 1253-60.

8 Gutierrez PL. The role of $\mathrm{NAD}(\mathrm{P}) \mathrm{H}$ oxidoreductase (DT-dia-phorase) in the bioactivation of quinone-containing antitumor agents. Free Radic Biol Med 2000; 29: 263-75.

9 Shadle SE, Bammel BP, Cusack BJ, Knighton RA, Olson SJ, Mushlin PS, et al. Daunorubicin cardiotoxicity: evidence for the importance of the quinine moiety in a free-radical-independent mechanism. Biochem Pharmacol 2000; 60: 1435-44.

10 Wang L, Ma W, Markovich R, Chen JW, Wang PH. Regulation of cardiomyocyte apoptotic signaling by insulin-like growth factor I. Circ Res 1998; 83: 516-22.

11 Arola OJ, Saraste A, Pulkki K, Kallajoki M, Parvinen M, Voipio-Pulkki LM. Acute doxorubicin cardiotoxicity involves cardiomocyte apoptosis. Cancer Res 2000; 60: 1789-92.

12 Orrenius S. Mitochondrial regulation of apoptotic cell death. Toxicol Lett 2004; 149: 19-23.

13 Wu S, Ko YS, Teng MS, Ko YL, Hsu LA, Hsueh C, et al. Adriamycininduced cardiomyocyte and endothelial cell apoptosis: in vitro and in vivo studies. J Mol Cell Cardiol 2002; 34: 1595-607.

14 Green PS, Leeuwenburgh C. Mitochondrial dysfunction is an early indicator of doxorubicin-induced apoptosis. Biochim Biophys Acta 2002; 1588: 94-101.

15 Chen Y, Wang M, Rosen RT, Ho CT. 2,2-Diphenyl-1-picrylhydrayl radical-scavenging active components from Polygonum multiflorum thunb. J Agric Food Chem 1999; 47: 2226-8.

16 Chu J, Ye CF, Li L. Effects of stilbene-glycoside on learning and memory function and free radicals metabolism in dementia model mice. Chin J Rehabil Theory Practice 2003; 9: 643-5.

17 Liu L, Zhao L, Li YL, Zhang L, Ye CF, Li L. Protective effect of 2,3,5, 4'tetrahydroxystilbene-2- $\beta-0-D$-glucoside on hippocampal neurons in dementia rats induced by chronic cerebral ischemia. Chin J Pharm 2006; 41: 354-7.

18 Ban Y, Liu QL, Jin Y. Determination of stilbene-glucoside and investigation on its stability. Chin Trad Herb Drug 2004; 18: 1235-7.

19 Wang X, Zhao L, Han T, Chen S, Wang J. Protective effects of 2,3,5,4' tetrahydroxystilbene-2-O-beta- $D$-glucoside, an active component of Polygonum multiflorum Thunb, on experimental colitis in mice. Eur J Pharmacol 2008; 578: 339-48.

20 Estevez MD, Wolf A, Schramm U. Effect of PSC 833, verapamil and amiodarone on adriamycin toxicity in cultured rat cardiomyocytes. Toxicol in Vitro 2000; 14: 17-23.

21 Outomuro D, Grana DR, Azzato F, Milei J. Adriamycin-induced myocardial toxicity: new solutions for an ole problems? Int J Cardiol 2007; 117: 6-15.

22 Piscitelli SC, Rodvold KA, Rushing DA, Tewksbury DA. Pharmacokinetics and pharmacodynamics of doxorubicin in patients with small-cell lung cancer. Clin Pharmacol Ther 1993; 53: 555-61.

23 Li T, Danelisen I, Singal PK. Early changes in myocardial antioxidant enzymes in rats treated with adriamycin. Mol Cell Biochem 2002; 232: 19-26.

24 Wang L, Ma W, Markovich R, Lee WL, Wang PH. Insulin-like growth factor I mosulates induction of apoptotic signaling in H9C2 cardiac muscle cells. Endocrinology 1998; 139: 1354-60.

25 Kim Y, Ma AG, Kitta K, Fitch SN, Ikeda T, Ihara Y, et al. Anthracyclineinduced suppression of GATA-4 transcription factor: implication in the regulation of cardiac myocyte apoptosis. Mol Pharmacol 2003; 63: 368-77.

26 Kitta K, Day RM, Kim Y, Torregroza I, Evans T, Suzuki YJ. Hepatocyte growth factor induces GATA-4 phosphorylation and cell survival in cardiac muscle cells. J Biol Chem 2003; 278: 4705-12.

27 Sugawara T, Chan $\mathrm{PH}$. Reactive oxygen radicals and pathogenesis of neuronal death after cerebral ischemia. Antioxid Redox Signal 2003; 5: 597-607.

28 Yamada J, Yoshimura S, Yamakawa H, Sawada M, Nakagawa M, Hara $\mathrm{S}$, et al. Cell permeable ROS scavengers, Tiron and Tempol, rescue PC12 cell death caused by pyrogallol or hypoxia/reoxygenation. Neurosci Res 2003; 45: 1-8.

29 Zhuang S, Lynch MC, Kochecar IE. Caspase-8 mediated caspase-3 activation and cytochrome $c$ release during singlet oxygen-induced apoptosis of HL-60 cells. Exp Cell Res 1999; 250: 203-12.

30 Clementi ME, Giardina B, Di Stasio E, Mordente A, Misiti F. Doxorubicin-derived metabolites induce release of cytochrome $c$ and inhibition of respiration on cardiac isolated mitochondria. Anticancer Res 2003; 23: 2445-50.

31 Gille L, Nohl H. Analyses of the molecular mechanism of adriamycininduced cardiotoxicity. Free Radic Biol Med 1997; 23: 775-82.

32 Zorzato F, Sarviati G, Facchinetti T, Volpe P. Doxorubicin induces calcium release from terminal cisternae of skeletal muscle. J Cell Biol 1985; 99: 875-85.

33 Kim DH, Landry AB, Lee YS, Katz AM. Doxorubicin-induced calcium release from sarcoplasmic reticulum vesicles. J Moll Cell Cardiol 
1989; $21: 433-6$

34 Caroni P, Villani F, Carafoli E. The cardiotoxic antibiotic doxorubicin inhibits the $\mathrm{Na}^{+} / \mathrm{Ca}^{2+}$ exchange of dog heart sarcolemmal vesicles. FEBS Lett 1981; 30: 184-6.

35 Keung EC, Toll L, Ellis M, Jensen RA. L-type cardiac calcium channels in doxorubicin cardiomyopathy in rats morphological, biochemical, and functional correlations. J Clin Invest 1991; 87: 2108-13.

36 Przygodzki T, Sokal A, Bryszewska M. Calcium ionophore A 23187 action on cardiomyocytes is accompanied by enhanced production of reactive oxygen species. Biochim Biophys Acta 2005; 1740: 481-8.

37 Petrosillo G, Ruggiero F, Pistolese M, Paradies G. $\mathrm{Ca}^{2+}$-induced reactive oxygen species production promotes cytochrome $c$ release from rat liver mitochondria via mitochondirial permeability transition
(MPT)-dependent and MPT-independent mechanisms. J Biol Chem 2004; 279: 53103-8.

38 Waring P. Redox active calcium ion channels, cell death. Arch Biochem Biophys 2005; 434: 33-42.

39 Kim SY, Kim SJ, Kim BJ, Rah SY, Chung SM, Im MJ, et al. Doxorubicininduced reactive oxygen species generation and intracellular $\mathrm{Ca} 2+$ increase are reciprocally modulated in rat cardiomyocytes. Exp Mol Med 2006; 38: 535-45.

40 Kimura $\mathrm{Y}$, Okusa $\mathrm{H}$. Effects of naturally occurring stilbene glucosides from medicinal plants and wine, on tumor growth and lung metastasis in Lewis lung caraionma-bearing mice. J Pharm Pharmacol 2000; 52 : 1287-95. 